\title{
Expression of NANOG in human gliomas and its relationship with undifferentiated glioma cells
}

\author{
CHAO-SHI NIU ${ }^{1-3^{*}}$, DONG-XUE LI ${ }^{1,2^{*}}$, YU-HAI LIU ${ }^{1,2}$, XIAN-MING FU ${ }^{1-3}$, SHEN-FENG TANG ${ }^{2}$ and JING LI ${ }^{2}$ \\ ${ }^{1}$ Department of Neurosurgery, Anhui Provincial Hospital Affiliated to Anhui Medical University; ${ }^{2}$ Molecular Neurobiology \\ and Neural Regeneration and Repair Laboratory, Anhui Provincial Stereotactic Neurosurgical Institute; \\ ${ }^{3}$ Anhui Provincial Stereotactic Neurosurgical Institute, Hefei, Anhui 230001, P.R. China
}

Received February 22, 2011; Accepted April 14, 2011

DOI: $10.3892 /$ or.2011.1308

\begin{abstract}
Stemness genes, including NANOG, which have been reported to play a significant role in embryonic stem cells (ESCs), are purported to be expressed in specific human tumor types. In the present study, we explored the expression of NANOG in gliomas to demonstrate its key roles in maintaining the undifferentiated state of glioma cells. Brain tumor stem cells (BTSCs) were isolated from the human glioma cell line U87 and cultured in simplified serum-free medium. Significantly higher NANOG mRNA and protein expression levels were demonstrated in U87 parental attached cells and suspended BTSCs as well as in 69 glioma specimens of different pathological grades. The relative levels of NANOG mRNA and protein expression were higher in the BTSCs as compared to those in the U87 parental attached cells and were significantly positively correlated with pathological grade. The coexpression and relationship of NANOG, CD133 and GFAP in situ in the cellular levels was determined through double-label immunohistochemical staining in the gliomas. A positive correlation of NANOG and CD133 expression with pathological grade of the samples was noted, while NANOG and GFAP expression correlated negatively with the pathological grade $(\mathrm{P}<0.01)$. Overexpression of NANOG in gliomas and its close relationship with the undifferentiated state of glioma cells in vivo and in vitro indicated that NANOG may contribute to the existence of BTSCs and may be related to tumorigenesis of the cerebrum by maintaining the undifferentiated state of glioma cells, which provides a foundation to further explore its role in the biological behavior of gliomas.
\end{abstract}

Correspondence to: DrChao-Shi Niu,Department of Neurosurgery, Anhui Provincial Hospital, Anhui Medical University, Lujiang Road 17, Hefei, Anhui 230001, P.R. China

E-mail: niuchaoshi@126.com

${ }^{*}$ Contributed equally

Key words: glioma, NANOG, differentiation, expression

\section{Introduction}

Cancer cells and embryonic stem cells (ESCs) share many key biological properties, such as self-renewal, an undifferentiated state, extensive proliferative potency, pluripotency and differentiation capacity. These parallel features suggest that similar mechanisms may be involved in regulating cancer cells and ESCs. This has given rise to the hypothesis that cancer is a stem cell disease, and recently this concept has attracted much attention (1). The cancer stem cell (CSC) theory of tumorigenesis assumes the possibility of the identification of a small group of tumor cells responsible for the occurrence, growth, and recurrence of tumors in different types of cancers including gliomas (2-4). Therefore, CSCs are likely to be the most relevant targets in eradicating tumors, and further studies on the characterization of CSCs may contribute to a more effective treatment of tumors (5). One promising approach is to target core stemness genes which are well known for playing important roles in the biological properties of ESCs.

NANOG has a critical role in regulating the cell fate of the pluripotent inner cell mass during embryonic development, maintaining the pluripotent epiblast and preventing differentiation (6-8). Indeed, combined with OCT4 and SOX2, it is considered as a master gene of mammalian embryogenesis and forms a core ESC network that coordinately determines ESC self-renewal and differentiation (9). Overexpression of NANOG enables maintenance of these cells in an undifferentiated pluripotent state for an extended period, whereas inhibition of NANOG leads to the accelerated differentiation of ESCs (10). NANOG is also involved in the reprogramming of differentiated cells towards the induction of pluripotent stem (iPS) cells by reprogramming gene sets that include OCT4, SOX2, c-myc and Klf4 (11,12). These findings suggest that NANOG plays an important role in the self-renewal and undifferentiated pluripotency of ESCs. Additionally, previous studies have revealed that NANOG is detected not only in germ cell tumors (13-15) but also in several types of tumors and tumor cell lines including carcinomas of the cervix, breast, kidney, oral cavity, ovary, prostate and osteosarcoma cell lines (16-24). Furthermore, Santagata et al reported that NANOG is a sensitive and specific marker of central nervous system germinoma apart from gliomas (25). In contrast, Ben-Porath et al reported that gliomas express a core 
ES-like stem signature including NANOG (26). Subsequently, Zbinden et al addressed the function of NANOG in human glioblastomas and its relationship with HH-GLI activity (27). However, comprehensive and systematic studies of NANOG mRNA and protein expression in glioma cell lines in vitro and in distinct multiple glioma pathological types particularly low-grade gliomas in vivo as well as its relationship with other important glioma undifferentiation markers in situ in cellular levels are still lacking mainly considering the scarce sample data or the limitations in methodology.

In contrast, OCT4 was found to be overexpressed in human gliomas and promoted cell proliferation and colony formation in the C6 glioma cell line (5). SOX2 was found to be expressed in glioma tissue at an early and a progressed stage (28). Moreover, silencing of SOX2 resulted in inhibition of proliferation and loss of tumorigenicity in glioblastoma in immunodeficient mice, which suggests a hierarchical model of cerebral tumors controlled by SOX2 and opens a path to identify downstream genes as therapeutic targets (29).

We investigated whether NANOG is important in the tumorigenesis and malignant progression of gliomas and whether it contributes to the undifferentiated state of glioma cells. We preliminarily examined the expression of NANOG in the glioma cell line U87 and in glioma tissues of different pathological grades. We also investigated the expression relationship in situ between NANOG and the BTSC marker, CD133, and glioma differentiation marker, GFAP, to demonstrate a significant roles in maintaining the undifferentiated state of glioma cells.

\section{Materials and methods}

Clinical sample collection. Glioma samples were obtained from 69 patients with primary gliomas who underwent surgical treatment at the Department of Neurosurgery, Anhui Provincial Hospital, Anhui Medial University, between October 2008 and September 2009 in accordance with the National Regulation of Clinical Sampling in China. Adult normal brain tissues for use as normal controls were obtained from surgical resections of 7 trauma patients. Informed consent was obtained from all patients before surgery as advocated by the regional ethics committee. Tumor specimens were immediately sectioned from the resected glioma tissues, formalin-fixed and paraffin-embedded or frozen in liquid nitrogen, and stored at $-80^{\circ} \mathrm{C}$ until RNA/protein extraction. All of the glioma samples were verified by pathological analysis and classified according to the WHO 2007 classification standard. There were 26 low-grade (WHO Grade II) and 43 high-grade tumors (WHO Grades III and IV). None of the patients had received chemotherapy, immunotherapy and radiotherapy prior to specimen collection.

Cell culture. The U87 glioma cell line (Chinese Academy of Sciences Type Culture Collection) was grown as a monolayer of cells in Dulbecco's modified Eagle's medium:nutrient mixture F-12 (Ham's) (1:1) (DMEM/F-12) (Gibco, USA), supplemented with $10 \%$ fetal bovine serum (FBS) (Hyclone, USA), $100 \mathrm{U} / \mathrm{ml}$ penicillin/streptomycin (Gibco). For isolation of BTSCs from the U87 glioma cell line, U87 attached cells were dissociated and seeded at a density of $3 \times 10^{6}$ live cells/60-mm plate in simplified serum-free medium consisting of DMEM/F12, 2\% B27 supplement (Gibco), $20 \mu \mathrm{g} / 1$ human recombinant basic fibroblast growth factor (bFGF) (Gibco), $20 \mu \mathrm{g} / 1$ epidermal growth factor (EGF) (Gibco), $2 \mathrm{mmol} / \mathrm{l}$ L-glutamine, $4 \mathrm{U} / 1$ insulin and $100 \mathrm{U} / \mathrm{ml}$ penicillin/streptomycin (Gibco), pH 7.2-7.5. The medium was replaced every other day until primary tumor spheres were visible under microscopy within $\sim 2$ weeks.

After the primary tumor spheres reached the size of approximately 100-200 cells/sphere, the spheres were dissociated and plated in 96-well plates in $100 \mu \mathrm{l}$ volumes of simplified serum-free medium. Every 2 days, $20 \mu 1$ of simplified serum-free medium was added to the culture. The secondary spheres derived from single cells of the primary tumor spheres were dynamically observed under microscopy and used for subsequent assay. The stemness and differentiation properties were observed via immunocytochemical staining described subsequently as tumor spheres and differentiated cells after re-inoculation onto serum-containing medium at different times.

Immunocytochemistry and immunohistochemical staining. Standard immunocytochemical/immunohistochemical protocols were used. For immunocytochemistry, cells were plated on poly-D-lysine/laminin-coated glass coverslips for a few days, fixed with $4 \%$ paraformaldehyde, treated with PBS, and blocked with $10 \%$ goat (or rabbit) serum (Gibco). For cytoplasmic (and nuclear) proteins permeabilization was performed using $0.2 \%$ Triton $\mathrm{X}-100$ (10 min at room temperature). Consequently, coverslips were incubated with various primary antibodies at $4^{\circ} \mathrm{C}$ overnight, followed by FITCor TRITC-conjugated secondary antibodies and using the PV-9003 kit (Beijing Zhongshan Golden Bridge Biotechnology Co., Ltd. (ZSGB-Bio), China). The cells were counterstained with 4',6-diamidino-2-phenylindole (DAPI) (Sigma, USA) or hematoxylin to identify all nuclei. The images were acquired using an Olympus BX51 fluorescence microscope (Japan). The primary antibodies used were as follows: mouse monoclonal anti-CD133 (Miltenyi, Germany), rabbit polyclonal anti-GFAP, rabbit polyclonal anti-NSE (ZSGB-BIO) and rabbit polyclonal anti-NANOG antibody (R\&D Systems, USA).

For immunohistochemistry, formalin-fixed, paraffinembedded tissue sections $(4 \mu \mathrm{m})$ were deparaffinized and hydrated. Endogenous peroxidase activity was blocked (3\% $\mathrm{H}_{2} \mathrm{O}_{2}$ ), and antigen retrieval was performed $(10 \mathrm{mM}$ citrate buffer; $\mathrm{pH}$ 6.0). Incubation was carried out with $10 \%$ goat (or rabbit) serum for $10 \mathrm{~min}$ to block non-specific antibody binding. The sections were incubated with the various primary antibodies (described above) at $4^{\circ} \mathrm{C}$ overnight, followed by staining with the PV-9003 kit or SP-9002 kit (ZSGB-BIO). Immunopositive tumor cells were visualized using 3,3 diaminobenzidine (DAB) (Sigma). The sections were lightly counterstained with hematoxylin. Adjacent sections were used as controls. Two pathologists scoring the immunohistochemical staining were blinded to the clinical data. Images captured for all sections were acquired using an Olympus BX51. Interpretation of staining was carried out in 10 high-power views (x400 objective) for each slide. Positive cells were indicated by the presence of a distinct brown color in the nuclei or cytoplasm. In the negative controls, 
the antibodies were replaced by PBS and then reacted as described above.

Double-label immunohistochemical staining. For NANOG immunohistochemical staining, the same protocol as described above was used. After detection of NANOG using the SABC-AP kit (Boster, Wuhan, China) and BCIP/NBT (Boster), the sections were processed $(10 \mathrm{mM}$ citrate buffer; $\mathrm{pH} 6.0)$ for $2 \mathrm{~min}$, and then incubated with antibodies against either the intermediate filament protein marker CD133 or GFAP followed by the secondary antibody SP-9002 or SP-9001 and visualized using DAB. Adjacent sections were single stained immunohistochemically for NANOG, CD133 and GFAP in order to validate the immunohistochemical staining. Semiquantification of cells positive for NANOG, CD133, GFAP, NANOG/CD133 and NANOG/GFAP was carried out by counting all of the stained cells within 10 randomly selected microscope fields per coverslip, and the percentage was calculated based on the total number of nuclei counted.

$R T-P C R$. Total RNA was extracted from the cultured cells or the human glioma samples with TRIzol reagent (Invitrogen, USA) and treated with DNase (Fermentas, Vilnius, Lithuania) to remove DNA contamination. RNA (200 ng to $1 \mu \mathrm{g})$ and M-MLV (Takara, Japan) and oligo-dT (Takara) were used for cDNA synthesis. PCR was performed with $2 \mathrm{X}$ Taq Plus PCR MasterMix (Tiangen, China). The primer sequences and the size of the amplified product were as follows: NANOG (403 bp), 5'-ATGCCTGTGATTTGTGGGCC-3' (forward) and 5'-GCCAGTTGTTTTTCTGCCAC-3' (reverse); $\beta$-actin (186 bp), 5'-TGGCACCCAGCACAATGAA-3' (forward) and 5'-CTAAGTCATAGTCCGCCTAGAAGCA-3' (reverse). $\beta$-actin was used as the internal control. In semi-quantitative RT-PCR, standardized template amounts were used to amplify cDNA for 30-35 cycles. The PCR products were separated on $1.2 \%$ agarose gels by electrophoresis. The intensity of the bands was determined using the Quantity One software (USA).

Western blot analysis. Cells and tissues were washed with cold PBS and lysed in ice-cold RIPA buffer containing protease inhibitors (Beyotime Institute of Biotechnology, China). Cell lysates were incubated on ice for $30 \mathrm{~min}$ and then centrifuged at $4^{\circ} \mathrm{C}$ for $30 \mathrm{~min}$. The supernatant was collected, and the protein concentration was measured. Equivalent amounts of protein in each sample were separated on a $10 \%$ SDS-polyacrylamide gel electrophoresis (Beyotime Institute of Biotechnology) and then electrotransferred to a PVDF membrane (Millipore, USA) and blocked. Membranes were then probed with goat polyclonal anti-NANOG antibody (R\&D Systems, $1: 50$ ) at $4^{\circ} \mathrm{C}$ overnight or mouse monoclonal anti- $\beta$-actin antibody (Beyotime, $1: 1000$ ) for $1 \mathrm{~h}$ at room temperature followed by the horseradish peroxidase (HRP)conjugated rabbit anti-goat or goat anti-mouse IgG antibody (ZSGB-BIO). Immunoblots were visualized by chemiluminescence using an ECL detection system (BeyoECL Plus, Beyotime, China). The intensity of the bands was determined using the Image-Pro Plus 6.0 software (Japan).

Statistical analysis. Statistical Package for the Social Sciences software (version 17.0) was used for statistical analysis. Data
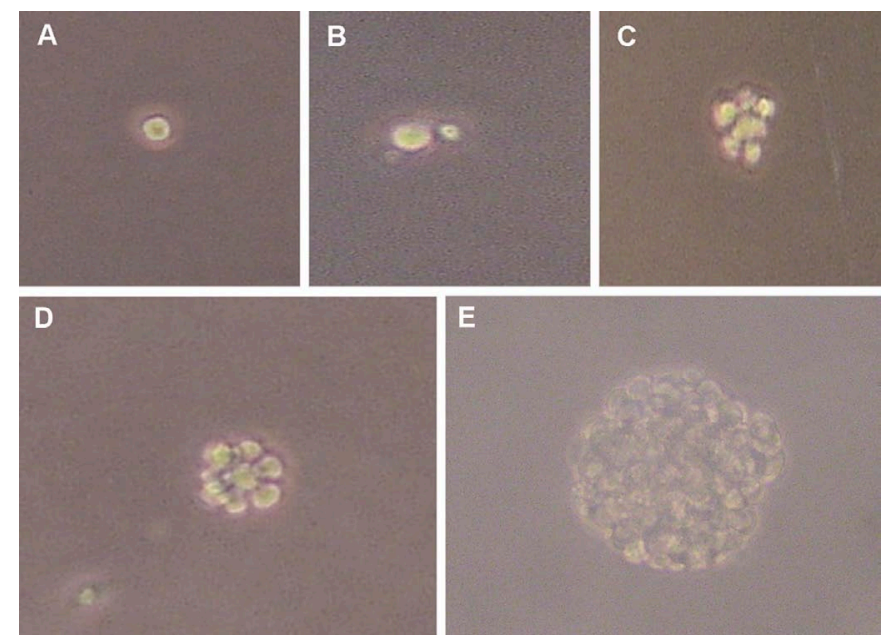

Figure 1. Secondary spheres derived from single cells of the primary tumor spheres (magnification, x400). (A) One hour, (B) $12 \mathrm{~h}$ and (C) $36 \mathrm{~h}$ after inoculation. (D) Five days after inoculation. (E) One to 2 weeks after inoculation.

in the text and figures were expressed as the mean \pm SD. The independent Student's t-test or one-way analysis of variance (ANOVA) was used to compare the expression level of NANOG between groups. Correlation analysis of the expression levels of NANOG, CD133 and GFAP was performed using the Spearman rank-sum test. The level of statistical significance was set at $\leq 0.05$ for all tests.

\section{Results}

Human glioma cell line U87 contains BTSCs. U87 cells showed suspended or semi-suspended growth. A portion of the cells were adherent in simplified serum-free medium and a small fraction of cells demonstrated growth into clonally derived neurosphere-like clusters observed within 24-48 h. Tumor spheres continued to proliferate and expand over time. Within 2 weeks, the diameter of these spheres increased by 10 - to 20 -fold. A single-cell suspension prepared from tumor spheres was examined for its capacity to form secondary spheres by single parental cells in fresh simplified serum-free medium. The secondary spheres, which were round or oval, bright and uniform in structure, were formed in the wells within 1-2 weeks (Fig. 1). Tumor spheres were passaged every 2-3 weeks for many generations in fresh simplified serum-free medium.

CD133, a cell surface marker for putative neural stem cells (NSCs), is widely accepted as a markers for BTSCs. The tumor secondary spheres cultured in DMEM/F12 with 10\% FBS for $4 \mathrm{~h}$ were positive for CD133 (Fig. 2A-C). To assess the multi-directional differentiation ability of BTSCs, we tested their ability to differentiate upon removal of growth factors and addition of $10 \%$ FBS for 10 days. The cells from the tumor secondary spheres showed some degree of differentiation, clearly heterogeneous cell morphology, different nuclear sizes and were stained positive for GFAP and NSE (Fig. 2D-I). Expression of GFAP and NSE was also analyzed as markers of glial differentiation and neuronal differentiation, respectively. 

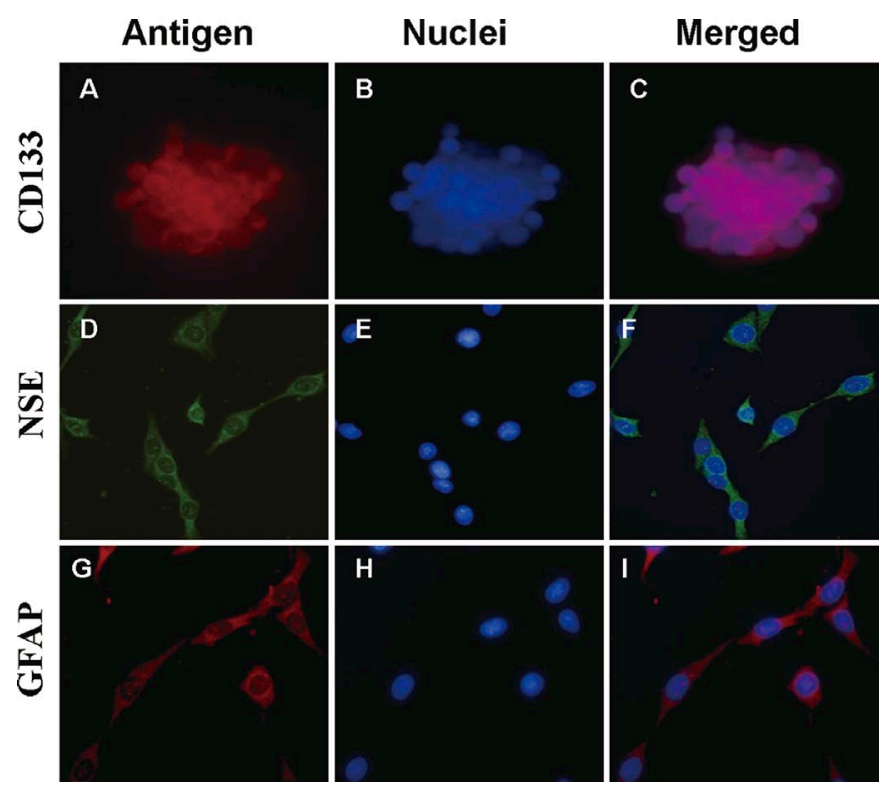

Figure 2. Expression of neural stem cell and differentiation markers by immunocytochemical staining (magnification, $\mathrm{x} 400$ ). (A) CD133 (TRITC); (D) NSE (FITC); (G) GFAP (TRITC); (B, E and H) nuclei (DAPI); (C, F and I) merged view.
NANOG was expressed in the U87 glioma cell line and enriched in BTSCs. NANOG expression was examined using RT-PCR, immunocytochemistry and Western blotting in the U87 attached cells and BTSCs. Immunocytochemical analysis revealed that NANOG protein was localized within the nuclei of the U87 attached cells and BTSCs (Fig. 3A and B). Western blotting confirmed that the protein level of NANOG in the BTSCs was also up-regulated compared with that of the U87 attached cells (Fig. 3C and E). Finally, RT-PCR analysis of cells revealed the expected 403-bp NANOG band, 186-bp $\beta$-actin band and an $\sim 1.5$-fold enrichment of NANOG mRNA in the BTSCs as compared with the U87 attached cells $(\mathrm{t}=4.770, \mathrm{P}<0.01)$ (Fig. 3D and $\mathrm{F}$ ). No obvious band was observed in the negative and blank control.

NANOG was highly expressed in human gliomas and positively correlated with pathological grade. We proceeded to analyze the expression profiles of 69 gliomas to examine whether NANOG was enriched in glioma tissues. To investigate whether NANOG could be transcribed, we initially used the primers described above. As expected, the results revealed the expected NANOG band in the glioma tissues of different pathological grade (Fig. 4J). Densitometric evaluation of the relative expression showed that the mRNA level of NANOG
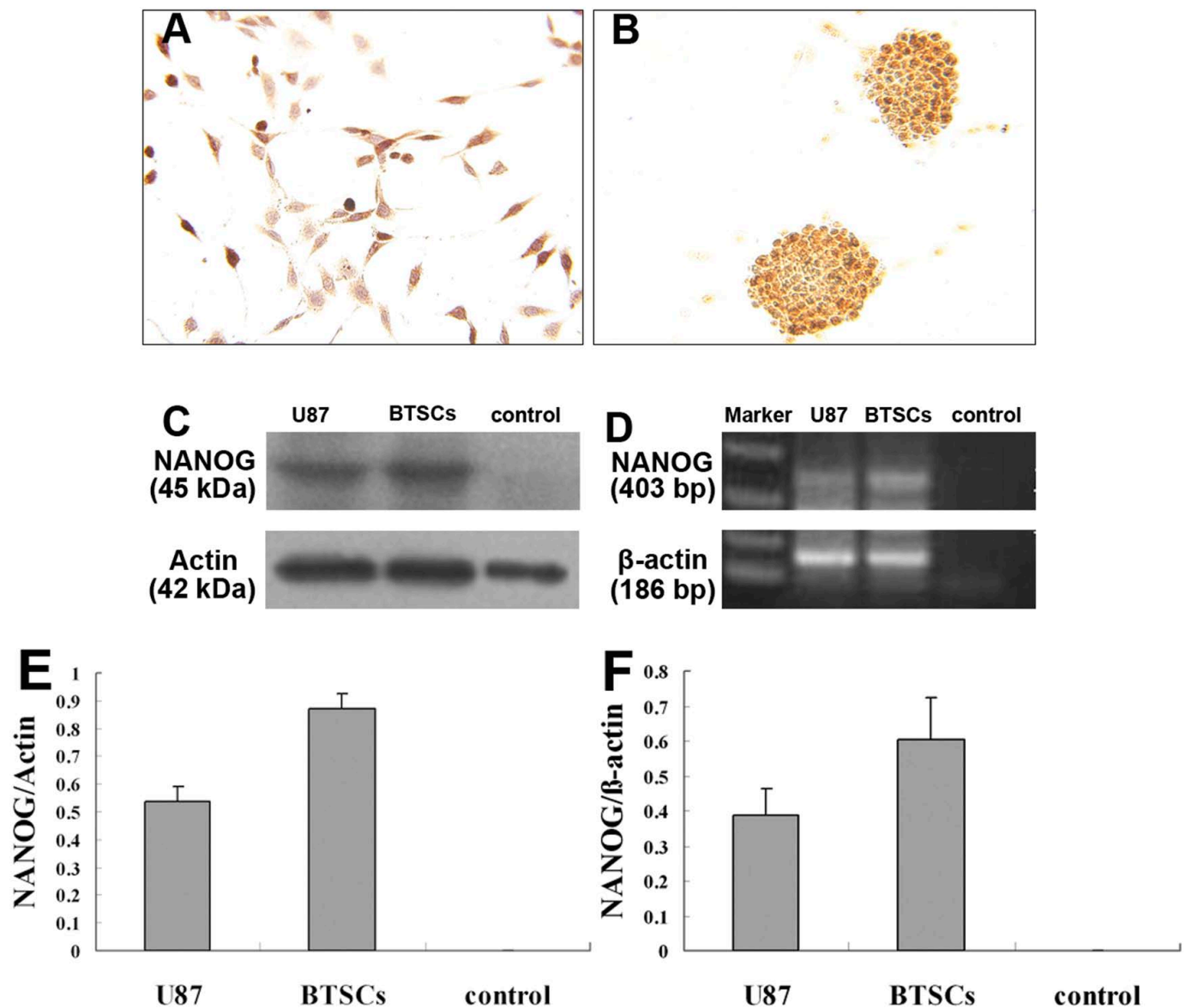

Figure 3. Expression of NANOG in U87 parental attached cells and BTSCs in vitro. NANOG immunocytochemical staining in (A) U87 parental attached cells (magnification, $\mathrm{x} 400$ ) and (B) BTSCs (magnification, x400). (C) Western blot analysis of U87 parental attached cells and BTSCs (normal brain tissues as control). (E) Histogram representing the relative level of NANOG protein as determined by Western blot analysis $(\mathrm{P}<0.01$, independent Student's $\mathrm{t}$-test). (D) Expression of NANOG mRNA as determined by RT-PCR in U87 parental attached cells and BTSCs (removing the template as control). (F) Histogram representing the relative level of NANOG mRNA as determined by RT-PCR $(\mathrm{P}<0.01$, independent Student's $\mathrm{t}$-test). 

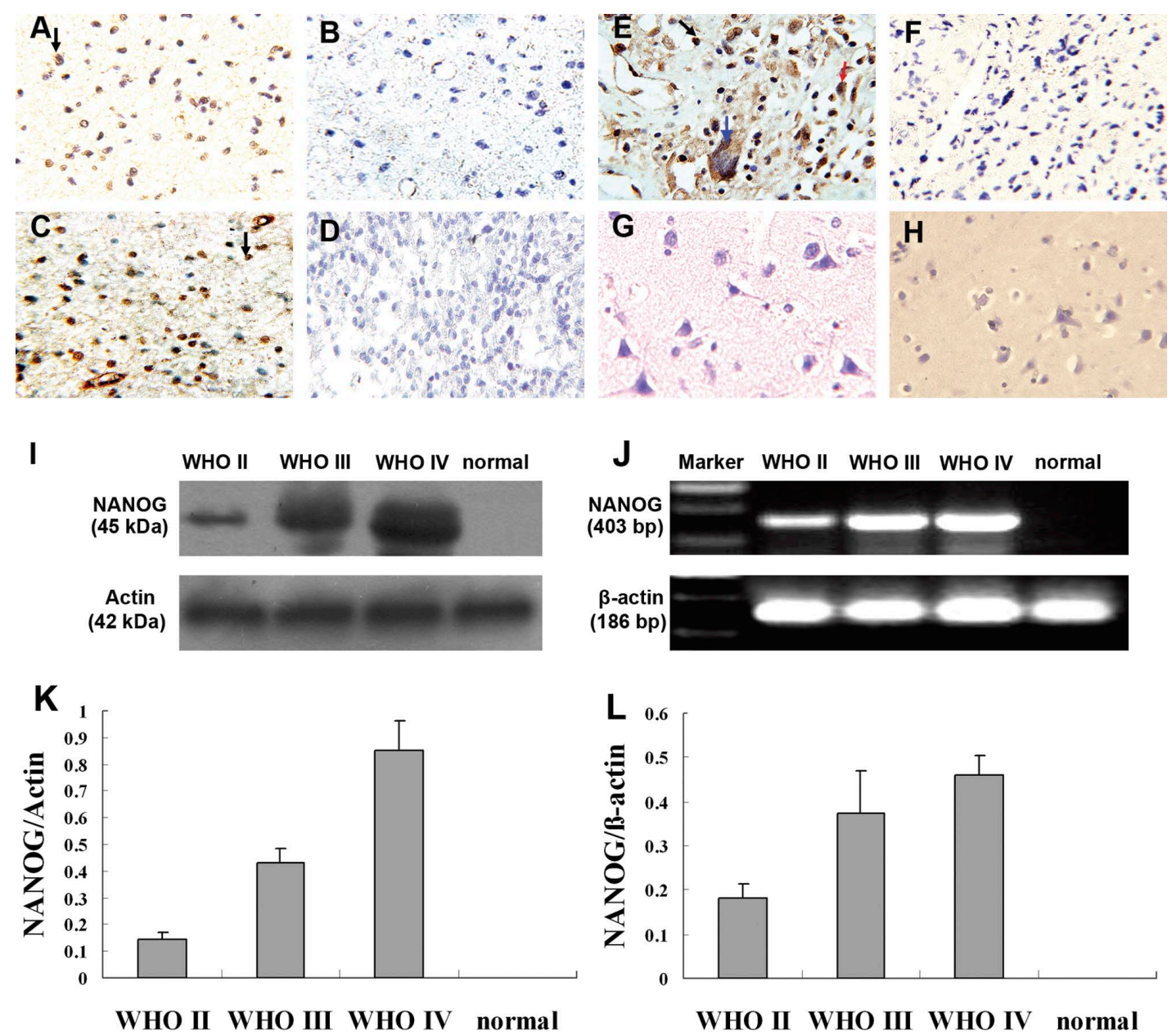

Figure 4. Expression of NANOG in differential pathological grade glioma tissues in vivo. (A-H) NANOG immunohistochemical staining of paraffin sections of gliomas (magnification, $x 400$ ). (A) Expression of NANOG in WHO Grade II tissue; NANOG expression was primarily localized in the nuclei of tumor cells (black arrow). (C) Expression of NANOG in WHO Grade III tissue; NANOG expression was primarily localized in the nuclei of tumor cells (black arrow). (E) Expression of NANOG in WHO Grade IV tissue; NANOG expression was primarily localized in the nuclei of tumor cells (black arrow), particularly tumor giant cells (blue arrow). However, cytoplasmic staining was also observed (red arrow). (G) Normal brain tissue. (B, D, F and H) Negative control using corresponding adjacent sections. (I) Western blot analysis of different pathological grade glioma tissues (normal brain tissues as control). (K) Histogram representing the relative level of NANOG protein as determined by Western blot analysis (P<0.01, ANOVA). (J) Expression of NANOG mRNA by RT-PCR in different pathological grade glioma tissues (normal brain tissues as control). (L) Histogram representing relative level of NANOG mRNA by RT-PCR $(\mathrm{P}<0.01, \mathrm{ANOVA})$

in the high-grade primary gliomas was significantly higher than that in in the low-grade gliomas $(\mathrm{F}=65.901, \mathrm{P}<0.01)$ (Fig. 4L). Meanwhile, the immunohistochemical analysis showed that NANOG was expressed in all of the 69 human glioma samples albeit at different protein levels. Furthermore, a marked postive correlation was noted between pathological grade and the expression of NANOG $(\mathrm{F}=50.86, \mathrm{P}<0.01)$. WHO III-IV grade glioma tissues, which represent the most aggressive subtype of glioma, showed significant NANOG enrichment and moderate or strong expression, in contrast to a lower degree of enrichment and weak expression in WHO I grade tissues. By contrast, no obvious expression was observed in the 7 normal brain samples and their respective blank controls (Fig. 4A-H). Following the above observations, we carried out Western blot analysis of NANOG to confirm the relationship between the expression of NANOG and pathological grade. A similar differential expression pattern was noted as elevated NANOG expression levels were also correlated with increasing degrees of pathological grade
(Fig. 4I). These results indicate that NANOG is preferentially overexpressed in poorly differentiated gliomas. However, similar to the histopathology of gliomas, NANOG expression was heterogeneous at both intertumoral and intratumoral levels. In addition, human NANOG has 305 aa anticipated to encode an $\sim 35-\mathrm{kD}$ a protein, while NANOG protein of $\sim 45 \mathrm{kDa}$ was detected in the gliomas by Western blot analysis.

NANOG protein was mainly localized in the nuclei of human glioma cells. Human NANOG is a transcriptional regulator and is localized in the nucleus. In human glioma cell line U87, the immunohistochemical analysis showed that both U87 attached cells and BTSCs were positive for NANOG, and the expression was mainly localized in the nuclei of these cells (Fig. 3A and B). The immunohistochemical analysis showed that NANOG protein, in the majority of cases, was localized in the nuclei of tumor cells. The nuclei of most high-grade glioma cells were more intensely stained compared with those of low-grade tumors (Fig. 4A-H), indicating that NANOG was 

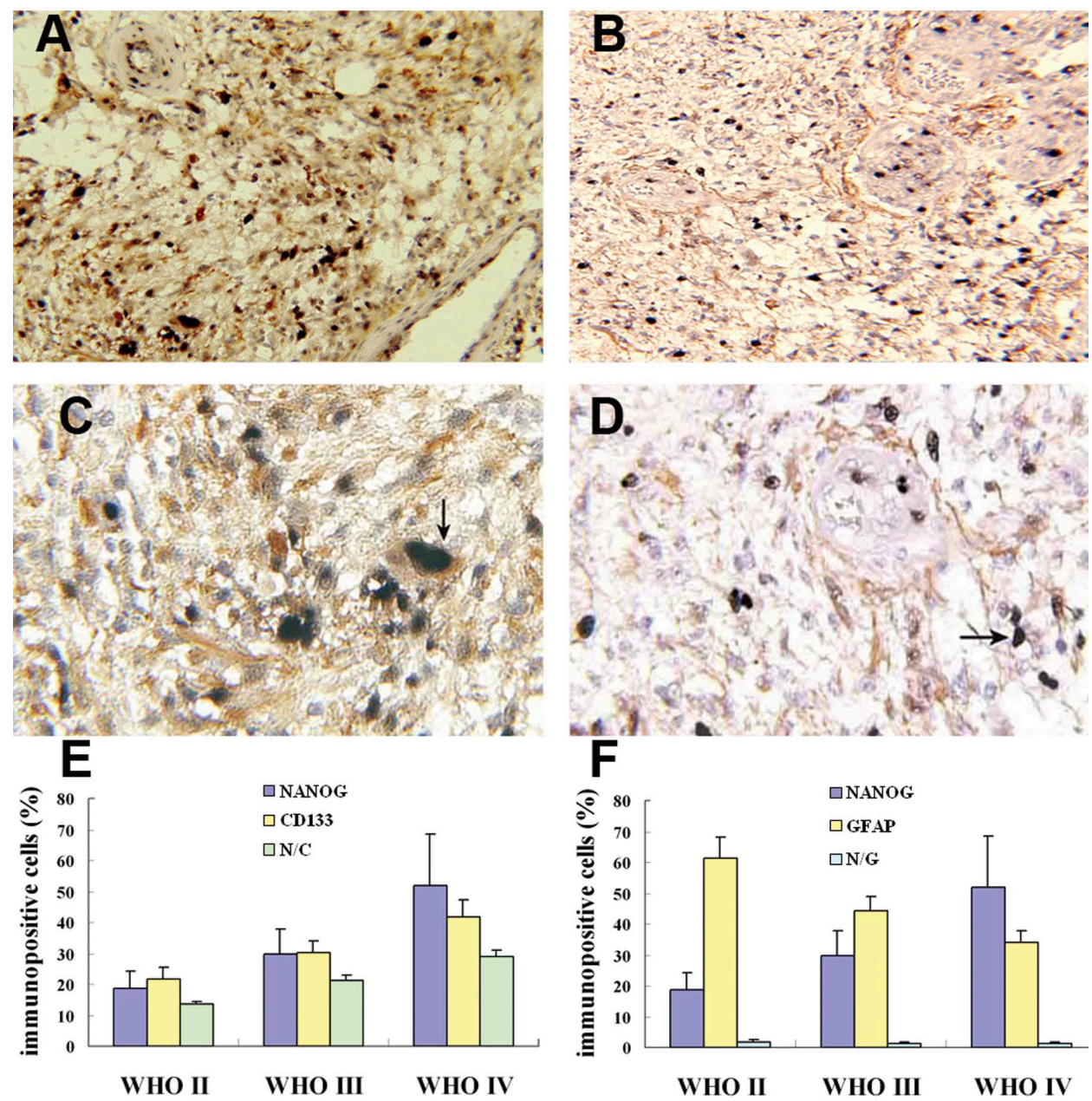

Figure 5. Coexpression of NANOG/CD133 and NANOG/GFAP by double-label immunohistochemical staining. (A) Coexpression of NANOG/CD133 in gliomas (magnification, x200). (B) Coexpression of NANOG/GFAP in gliomas (magnification, x200). (C) Coexpression of NANOG/CD133 in gliomas. $\mathrm{NANOG}^{+} / \mathrm{CD} 133^{+}$glioma cells were observed (black arrow) (magnification, x400). (D) Coexpression of NANOG/GFAP in gliomas. NANOG $/ \mathrm{GFAP}^{-}$glioma cells were observed (black arrow) (magnification, $\mathrm{x} 400$ ). (E) Histogram representing relative expression level of NANOG and CD133 in gliomas (P<0.01, ANOVA and Spearman rank-sum test). (F) Histogram representing the relative expression level of NANOG and GFAP in the gliomas (P<0.01, ANOVA and Spearman rank-sum test). N/C, NANOG/CD133. N/G, NANOG/GFAP.

expressed in the nuclei of glioma cells in a grade-dependent manner. However, cytoplasmic staining was observed in a few cases (Fig. 4E).

Coexpression and relationship of NANOG, CD133 and GFAP in gliomas. To confirm the possibility that NANOG contributes to maintenance of the undifferentiated state of glioma cells, we initially conducted double-label immunohistochemical staining for NANOG and CD133. The results revealed that the $\mathrm{CD} 133^{+}$BTSCs appeared to express higher levels of NANOG (Fig. 5A and C). $\mathrm{CD}^{2} 33^{+}$domain was enriched in the dense areas with $\mathrm{NANOG}^{+}$expression or vice versa. CD133 showed typical plasma membrane and cytoplasmic staining, whereas NANOG showed mainly nuclear or perinuclear staining with some cytoplasmic localization. Dual staining of $\mathrm{NANOG}^{+} / \mathrm{CD} 33^{+}$indicated that NANOG protein was colocalized within the nuclei of $\mathrm{CD} 133^{+}$glioma cells; however, not every $\mathrm{CD} 133^{+}$-stained cell was costained with NANOG (Fig. 5A and C). Moreover, we frequently observed an inverse relationship between NANOG and the differentiation marker GFAP. GFAP ${ }^{+}$domain was enriched in the less dense areas exhibiting $\mathrm{NANOG}^{+}$expression or vice versa. GFAP showed typical plasma membrane and cytoplasmic localization (Fig. 5B and D). The percentage of $\mathrm{NANOG}^{+}$ ( $\mathrm{F}=50.86, \mathrm{P}<0.01)$ (Fig. 5), $\mathrm{CD} 133^{+}(\mathrm{F}=131.4, \mathrm{P}<0.01)$ (Fig. 5A, $\mathrm{C}$ and $\mathrm{E})$, $\operatorname{GFAP}^{+}(\mathrm{F}=196.5, \mathrm{P}<0.01)$ (Fig. 5B, D and $\left.\mathrm{F}\right)$ and $\mathrm{NANOG}^{+} / \mathrm{CD} 133^{+}(\mathrm{F}=610.9, \mathrm{P}<0.01)$ (Fig. 5A, C and $\left.\mathrm{E}\right)$ cells was significantly varied in the different pathological grades of the human gliomas, apart from the $\mathrm{NANOG}^{+} / \mathrm{GFAP}^{+}$ cells $(\mathrm{F}=2.18, \mathrm{P}>0.1)$ (Fig. 5B, D and F). The expression levels of $\mathrm{NANOG}^{+}$and $\mathrm{CD} 133^{+}$were positively correlated $(\mathrm{r}=0.774, \mathrm{P}<0.01)\left(\right.$ Fig. 5E), while levels of $\mathrm{NANOG}^{+}$and $\mathrm{GFAP}^{+}$expression were negatively related $(\mathrm{r}=-0.770, \mathrm{P}<0.01)$ (Fig. 5F). Futhermore, there existed a positive correlation in the expression of the $\mathrm{NANOG}^{+} / \mathrm{CD} 133^{+}$and $\mathrm{CD} 133^{+}$cells $(\mathrm{r}=0.864, \mathrm{P}<0.01)($ Fig. 5E).

\section{Discussion}

Glioma, a devastating invasive cerebral tumor, is the leading cause of central nervous system tumor-related death. Although major advances have been made in surgery, chemotherapy and 
radiotherapy for gliomas, the prognosis of glioma patients remains poor. To date, the pathogenesis of gliomas remains undetermined. The CSC hypothesis suggests that cancer occurrence, growth, evolution, metastasis and recurrence after various therapies is due to a small fraction of cells possessing the capacity for self-renewal, an undifferentiation state, infinite proliferation and tumorigenicity. The first direct evidence for CSCs was the discovery that leukemia was found to originate in hematopoietic stem cells (30). The CSC hypothesis that represents a paradigm shift in our understanding of carcinogenesis and tumor cell biology behavior has fundamental implications for cancer risk assessment, early detection, prognostics and prevention (31). Recently, gliomas have also been found to contain a subpopulation of stem cell-like glioma cells, termed BTSCs, which plays a decisive role in the the initiation and progression of gliomas and provides a new pathway for the treatment of gliomas $(2,32,33)$.

Consistent with the above hypothesis, malignant tumor cells often express stemness genes, such as NANOG, OCT4 and SOX2, and activation targets of these genes have been shown to be overexpressed in histologically poorly differentiated tumors associated with dismal clinical outcome (26). This suggests that the existence of CSCs in many poorly differentiated tumors may be attributed to abnormal activation of these genes. Therefore, analysis of the expression and significance of these genes in gliomas may aid in the understanding of the tumorigenesis and progression of gliomas. The significant role of OCT4 and SOX2 in gliomas has been reported $(5,29)$. NANOG is a key stemness gene that is crucial for maintaining the biological properties of ESCs (34). Expression of NANOG is restricted to pluripotent cells, and its level decreases with the onset of differentiation and loss of pluripotency in these cells (7). Recently, it was reported that NANOG choreographs the synthesis of the naive epiblast ground state in the embryo and that this function is recapitulated in the culmination of somatic cell reprogramming (35). Additionally, recent studies have shown that higher NANOG expression correlates with tumor progression, malignancy, and prognosis in prostate cancer and oral squamous cell carcinoma $(20,23)$.

We initially determined the expression of NANOG in long-term cultured gliomas cells in vitro. U87 is a classic glioma cell line. The results indicated that NANOG was highly expressed in the nuclei of U87 cells. Furthermore, the expression level of NANOG in enriched BTSCs was also up-regulated compared with those of U87 attached cells. This finding corroborates the results of Zbinden et al who showed near ubiquitous expression of NANOG protein in U87 cells (27). Yet, the different expression level of NANOG may be due to preferential recognization at varying levels of expression by the different antibodies. In addition, a possible reason that the actual NANOG band size was different from that predicted indicates post-translational modification, splice variants and multimers. On the other hand, as the genotype, phenotype and biological characteristics of U87 might be altered during long-term culture in serum-containing conditions, the existence of BTSCs in U87 remains controversial $(36,37)$. In the present study, isolation of BTSCs from U87 glioma cell was successfully carried out through methodologies based on the properties of BTSCs as follows. The tumor sphere reacted positively to CD133, a relatively specific cell surface marker of BTSCs. The sphere body formation of BTSCs was enriched by progressively increasing the concentration of simplified serum-free medium where the culture condition helps maintain the CSC undifferentiated state and the multi-directional differentiation ability upon removal of growth factors and addition of serum.

We next investigated the expression of NANOG in glioma tissues of differential pathological grade in vivo, which more realistically reflected the relationship between NANOG and gliomas. We found significantly higher NANOG mRNA and protein expression in glioma tissues as compared with the normal brain tissues. An association between higher NANOG expression and aggressive grades of gliomas was also demonstrated. This finding suggests that NANOG may participate in the pathogenesis of gliomas, which are defined as poorly differentiated according to purely histopathological criteria. Our results are consistent with Ben-Porath et al who reported that the expression level of NANOG was higher in poorly differentiated tumors, such as glioblastomas, breast cancer, and bladder cancer compared to that in well-differentiated tumors (26). The differential pathological grade of gliomas is assessed routinely in the clinic, with poorly differentiated gliomas generally exhibiting the worst prognoses. However, this classification is based on histopathological criteria with a poor description of the underlying molecular mechanism controlling glioma differentiation. Our analysis demonstrated an inverse relationship between the presence of enrichment of NANOG in gliomas and the degree of glioma differentiation. The conclusion is noteworthy. We suggest that the degree of glioma malignancy and differentiation is defined by the expression level of NANOG in combination with other conventional factors and, in addition, that the activity of NANOG contributes to generating stem-like phenotypes of poorly differentiated aggressive gliomas.

We also investigated the role of NANOG in anti-differentiation in gliomas by double-label immunohistochemical staining to detect the coexpression of NANOG/CD133 and NANOG/GFAP. Methodologically, we selected two immune enzyme systems. The primary antibodies were from different species, and the reaction products exhibited different colors. Subcellular localization was related but not overlapping and we could observe the correlation distribution of two proteins in situ. CD133 and GFAP are BTSC and glioma differentiation markers, respectively. In the present study, in areas with overexpression of NANOG, the expression level of CD133 was increased; whereas GFAP was decreased. Positive correlations of NANOG and CD133 expression and negative correlations of NANOG and GFAP expression with the different pathological grades in the glioma tissues were observed. This implies that NANOG may inhibit the differentiation of glioma cells to expand stem-like cells in gliomas. This would also explain why the expression levels of NANOG were increased with increasing grades of gliomas, while the mechanism remains to be further analyzed.

In the present study, $\mathrm{NANOG}^{+} / \mathrm{CD}^{-3} 3^{-}$or $\mathrm{NANOG}^{-} /$ $\mathrm{CD}_{133}{ }^{+}$cells were found by double-label immunohistochemistry. Therefore, we cannot definitely conclude that the NANOG-expressing cells were BTSCs. This method labeled not only BTSCs but also the intermediate cells or asymmetric division of precursor cells of the gliomas. Shemlkov et al 
reported that $\mathrm{CD} 133$ expression is not restricted to stem cells, and both $\mathrm{CD}_{133^{+}}$and $\mathrm{CD} 133^{-}$metastatic colon cancer cells initiate tumors (38). However, there is as yet no adequate stem cell marker for gliomas. The identification of BTSCs may be more accurate using multiple stem cell markers, and the final appraisal of BTSCs relies on the isolation of BTSCs, precise clone formation test in vitro and tumor growth test in vivo. What we can be certain is that there are two groups of BTSCs expressing NANOG or not.

In a previous study, NANOG was found to be localized in the nucleus (15). In the present study, we repeated the assay to exclude the possibility of false-positive staining and confirmed that NANOG protein was partly localized to the cytoplasm. The mechanism by which localization occurs is unknown, and further study is required. These findings were consistent with previous results which found that the NANOG protein was located in both the nuclei and in the cytoplasm of breast carcinoma and prostate cancer cells $(17,18,23)$. Furthermore, it is known that NANOG protein contains a DNA recognition sequence, implying that NANOG might regulate transcription in nuclei and mitochondria. However, the exact mechanism of NANOG activity in gliomas remains to be identified. Moreover, in agreement with the heterogeneity of NANOG expression, it was found to be heterogeneous in prostate cancer cells (23), typically containing cells at various stages of differentiation, NANOG is expressed in a highly variable percentage of cells, in the range of $10-80 \%$ in different glioma samples.

In conclusion, our comprehensive analysis indicated that overexpression of NANOG appears to be intimately involved in the pathogenesis of gliomas, and the activity of NANOG contributes to maintaining an undifferentiated state of glioma cells. Our results may provide further evidence for the CSC theory. On the basis of these findings, we assume that inhibition of NANOG, important in the progression of glioma cell transformation, may block the tumorigenesis of gliomas, and targeting NANOG may be an approach to improve the therapeutic intervention for poorly differentiated gliomas. However, further study is required to determine the precise role of NANOG and the mechanism of NANOG transcriptional regulation in gliomas.

\section{Acknowledgements}

Grant support for this study was provided by the National Natural Science Foundation of China (\#30672166). We thank all of the surgeons at the Neurosurgery Department of Anhui Provincial Hospital affiliated with Anhui Medical University for assisting in the collection of the tumor samples.

\section{References}

1. Huntly BJ and Gilliland DG: Cancer biology: summing up cancer stem cells. Nature 435: 1169-1170, 2005.

2. Singh SK, Clarke ID, Terasaki M, et al: Identification of a cancer stem cell in human brain tumors. Cancer Res 63: 5821-5828, 2003.

3. Bertrand J, Begaud-Grimaud G, Bessette B, Verdier M, Battu S and Jauberteau MO: Cancer stem cells from human glioma cell line are resistant to Fas-induced apoptosis. Int J Oncol 34: 717-727, 2009.

4. Li GH, Chen ZT, Hu YD, et al: Autocrine factors sustain glioblastoma stem cell self-renewal. Oncol Rep 21: 419-424, 2009.
5. Du Z, Jia D, Liu S, et al: Oct4 is expressed in human gliomas and promotes colony formation in glioma cells. Glia 57: 724-733, 2009.

6. Mitsui K, Tokuzawa Y, Itoh H, et al: The homeoprotein Nanog is required for maintenance of pluripotency in mouse epiblast and ES cells. Cell 113: 631-642, 2003.

7. Chambers I, Colby D, Robertson M, et al: Functional expression cloning of Nanog, a pluripotency sustaining factor in embryonic stem cells. Cell 113: 643-655, 2003.

8. Hart AH, Hartley L, Ibrahim M and Robb L: Identification, cloning and expression analysis of the pluripotency promoting Nanog genes in mouse and human. Dev Dyn 230: 187-198, 2004.

9. Boyer LA, Lee TI, Cole MF, et al: Core transcriptional regulatory circuitry in human embryonic stem cells. Cell 122: 947-956, 2005.

10. Darr H, Mayshar Y and Benvenisty N: Overexpression of NANOG in human ES cells enables feeder-free growth while inducing primitive ectoderm features. Development 133: 1193-1201, 2006.

11. Takahashi $\mathrm{K}$ and Yamanaka S: Induction of pluripotent stem cells from mouse embryonic and adult fibroblast cultures by defined factors. Cell 126: 663-676, 2006.

12. Yu J, Vodyanik MA, Smuga-Otto K, et al: Induced pluripotent stem cell lines derived from human somatic cells. Science 318: 1917-1920, 2007.

13. Almstrup K, Hoei-Hansen CE, Wirkner U, et al: Embryonic stem cell-like features of testicular carcinoma in situ revealed by genome-wide gene expression profiling. Cancer Res 64: 4736-4743, 2004.

14. You JS, Kang JK, Seo DW, et al: Depletion of embryonic stem cell signature by histone deacetylase inhibitor in NCCIT cells: involvement of Nanog suppression. Cancer Res 69: 5716-5725, 2009.

15. Hart AH, Hartley L, Parker K, et al: The pluripotency homeobox gene NANOG is expressed in human germ cell tumors. Cancer 104: 2092-2098, 2005.

16. Fujii H, Honoki K, Tsujiuchi T, Kido A, Yoshitani K and Takakura Y: Sphere-forming stem-like cell populations with drug resistance in human sarcoma cell lines. Int J Oncol 34: 1381-1386, 2009.

17. Ye F, Zhou C, Cheng Q, Shen J and Chen H: Stem-cellabundant proteins Nanog, Nucleostemin and Musashil are highly expressed in malignant cervical epithelial cells. BMC Cancer 8: 108, 2008.

18. Ezeh UI, Turek PJ, Reijo RA and Clark AT: Human embryonic stem cell genes OCT4, NANOG, STELLAR, and GDF3 are expressed in both seminoma and breast carcinoma. Cancer 104: 2255-2265, 2005.

19. Alldridge L, Metodieva G, Greenwood C, et al: Proteome profiling of breast tumors by gel electrophoresis and nanoscale electrospray ionization mass spectrometry. J Proteome Res 7: 1458-1469, 2008

20. Chiou SH, Yu CC, Huang CY, et al: Positive correlations of Oct-4 and Nanog in oral cancer stem-like cells and high-grade oral squamous cell carcinoma. Clin Cancer Res 14: 4085-4095, 2008.

21. Bussolati B, Bruno S, Grange C, Ferrando U and Camussi G: Identification of a tumor-initiating stem cell population in human renal carcinomas. FASEB J 22: 3696-3705, 2008.

22. Zhang S, Balch C, Chan MW, et al: Identification and characterization of ovarian cancer-initiating cells from primary human tumors. Cancer Res 68: 4311-4320, 2008.

23. Jeter CR, Badeaux M, Choy G, et al: Functional evidence that the self-renewal gene NANOG regulates human tumor development. Stem Cells 27: 993-1005, 2009.

24. Wang L, Park P and Lin CY: Characterization of stem cell attributes in human osteosarcoma cell lines. Cancer Biol Ther 8: 543-552, 2009.

25. Santagata S, Hornick JL and Ligon KL: Comparative analysis of germ cell transcription factors in CNS germinoma reveals diagnostic utility of NANOG. Am J Surg Pathol 30: 1613-1618, 2006.

26. Ben-Porath I, Thomson MW, Carey VJ, et al: An embryonic stem cell-like gene expression signature in poorly differentiated aggressive human tumors. Nat Genet 40: 499-507, 2008.

27. Zbinden M, Duquet A, Lorente-Trigos A, Ngwabyt SN, Borges I and Ruiz i Altaba A: NANOG regulates glioma stem cells and is essential in vivo acting in a cross-functional network with GLI1 and p53. EMBO J 29: 2475-2476, 2010. 
28. Kong DS, Kim MH, Park WY, et al: The progression of gliomas is associated with cancer stem cell phenotype. Oncol Rep 19: 639-643, 2008

29. Gangemi RM, Griffero F, Marubbi D, et al: SOX2 silencing in glioblastoma tumor-initiating cells causes stop of proliferation and loss of tumorigenicity. Stem Cells 27: 40-48, 2009.

30. Lapidot T, Sirard C, Vormoor J, et al: A cell initiating human acute myeloid leukaemia after transplantation into SCID mice. Nature 367: 645-648, 1994.

31. Wicha MS, Liu S and Dontu G: Cancer stem cells: an old idea - a paradigm shift. Cancer Res 66: 1883-1890, 2006.

32. Singh SK, Clarke ID, Hide T and Dirks PB: Cancer stem cells in nervous system tumors. Oncogene 23: 7267-7273, 2004

33. Li GH, Wei H, Lv SQ, Ji H and Wang DL: Knockdown of STAT3 expression by RNAi suppresses growth and induces apoptosis and differentiation in glioblastoma stem cells. Int $\mathbf{J}$ Oncol 37: 103-110, 2010.
34. Niwa H, Ogawa K, Shimosato D and Adachi K: A parallel circuit of LIF signalling pathways maintains pluripotency of mouse ES cells. Nature 460: 118-122, 2009.

35. Silva J, Nichols J, Theunissen TW, et al: Nanog is the gateway to the pluripotent ground state. Cell 138: 722-737, 2009.

36. Hirschmann-Jax C, Foster AE, Wulf GG, et al: A distinct 'side population' of cells with high drug efflux capacity in human tumor cells. Proc Natl Acad Sci USA 101: 14228-14233, 2004.

37. Kang SK, Park JB and Cha SH: Multipotent, dedifferentiated cancer stem-like cells from brain gliomas. Stem Cell Dev 15: 423-435, 2006

38. Shmelkov SV, Butler JM, Hooper AT, et al: CD133 expression is not restricted to stem cells, and both $\mathrm{CD}_{133^{+}}$and $\mathrm{CD} 133$ metastatic colon cancer cells initiate tumors. J Clin Invest 118: 2111-2120, 2008 